\title{
Hiperinsulinismo neonatal persistente. Análisis del diagnóstico diferencial a propósito de dos casos clínicos
}

\author{
M Isabel Hernández C, M Isabel Hodgson B, \\ Andreina Cattani 0. \\ Persistent neonatal hyperinsulinism. \\ Report of two cases
}

Persistent neonatal hyperinsulinism is the most common cause of refractory hypoglycemia during the first year of life. Inadequate insulin secretion is associated to mutations of four different genes, that can be diagnosed to orient patient management. We report two patients: a female newborn that presented a hypoglycemia of $16 \mathrm{mg} / \mathrm{dl}$ two hours after birth, was subjected to a subtotal pancreatectomy that did not correct hypoglycemia, requiring a total pancreatectomy. Pathological study of the pancreas showed a focal adenomatous hyperplasia. At the present time, she is three years of age and maintains euglycemia with fractionated feeding. A male newborn that had seizures at 28 hours of life and a hypoglycemia of $15 \mathrm{mg} / \mathrm{dl}$ was detected. He was also subjected to a subtotal pancreatectomy, that did not correct hypoglycemia and had to be extended to a total pancreatectomy. At the present time, he is 3 years and 11 months of age and has a normal psychomotor development (Rev Méd Chile 2004; 132: 995-1000).

(Key Words: Endogenous hyperinsulism; Hyperinsulism; Hypoglicemia)

Recibido el 23 de abril, 2003. Aceptado en versión corregida el 25 de mayo, 2004.

Departamento de Pediatría, Pontificia Universidad Católica de Chile.

E hiperinsulinismo neonatal persistente (HPN) nia en el primer año de vida, con alto riesgo de neuroglucopenia y daño neurológico imeversible ${ }^{1,2}$. Su incidencia vańa entre $1 / 27.000$ y $1 / 50.000$ recién nacidos (RN), llegando hasta $1 / 3.000$ en zonas de gran consanguinidad ${ }^{3}$. En el HNP severo, el tratamiento médico generalmente es inefectivo y la cirugía es mandatoria ${ }^{1,4}$. El análisis histológico del páncreas, demuestra que existe una forma difusa y otra focal. Su diagnóstico diferencial tiene importantes implicancias,

Correspondencia a: Andreina Cattani O. Fax: 2065011. E mail: acattani@med.puc.cl tanto en la conducta terapéutica, como en el pronóstico ${ }^{1,6-8}$. Por otro lado, en los últimos años, se han hecho importantes avances en el conocimiento de las bases moleculares de esta entidad ${ }^{4}$.

A propósito de dos casos clínicos de hiperinsulinismo severo, se revisan los métodos diagnósticos, bases moleculares de la enfermedad y conducta terapéutica.

\section{Caso CĹ́nico 1}

Recién nacido macrosómico, sexo femenino, primer hijo de padres sanos, no consanguíneos. Peso: 4.980 
g, talla: $54 \mathrm{~cm}$, Apgar 9-10. A las 2 horas de vida, estando asintomático, se detectó glicemia de 16 $\mathrm{mg} / \mathrm{dl}$. Se inició terapia con glucosa intravenosa (iv), llegando a requerir cargas de hasta $14 \mathrm{mg} / \mathrm{kg} /$ min. El estudio demostró: glucosa: 39 mg\%, insulina, determinada por inmunoquimioluminiscencia (ICMA): 14,2 $\mu \mathrm{U} / \mathrm{mL}$ (VN: <2), cetonemia (-); el tamizaje metabólico (gases venosos, aminoacidemia, lactato, amonemia), hormona de crecimiento, TSH y cortisol fueron normales. La presencia de niveles detectables de insulina concomitante con hipoglicemia confirmó un hiperinsulinismo. Como persistió con altos requerimientos de glucosa iv para lograr euglicemia, se inició Octreotide ${ }^{\circledR}$ (análogo de somatostatina) $15 \mu \mathrm{g} / \mathrm{kg} /$ día subcutáneo, con buena respuesta inicial. Posteriormente, aumentaron progresivamente los requerimientos de glucosa, indicándose pancreatectomía subtotal, respetando la cabeza del páncreas; la biopsia reveló acinos pancreáticos con distribución y morfología normal. A pesar de la cirugía, persistió con hiperinsulinismo no controlable con manejo médico, por lo que se completó pancreatectomía $( \pm 100 \%)$. El estudio histológico e inmunohistoquímico informó hiperplasia adenomatosa focal. Después de cirugía y hasta el octavo mes, requirió insulina y enzimas pancreáticas. Actualmente, a la edad de 3 años, se mantiene euglicémica sólo con alimentación fraccionada, lo que estaría reflejando hiperplasia del escaso tejido pancreático remanente. Su desarrollo psicomotor es normal.

\section{CASO CLÍNICO 2}

RN hijo de padres sanos, no consanguíneos; de 37 semanas de gestación, peso: $3.670 \mathrm{~g}$, talla $51,5 \mathrm{~cm}$, grande para edad gestacional. A las $28 \mathrm{~h}$ de vida presentó dos episodios convulsivos, constatándose hipoglicemia severa $(15 \mathrm{mg} / \mathrm{dl})$ controlada sólo con cargas de glucosa iv de hasta $18 \mathrm{mg} / \mathrm{kg} / \mathrm{min}$ y alimentación enteral. El estudio demostró: glicemia de $27 \mathrm{mg} / \mathrm{dl}$, insulinemia (ICMA): $40,8 \mu \mathrm{U} / \mathrm{mL}$ y cetonemia negativa; el tamizaje metabólico, TSH, hormona de crecimiento y cortisol fueron normales. Se inició tratamiento con diazóxido hasta $25 \mathrm{mg} / \mathrm{kg} /$ día, a pesar de lo cual persistió con altos requerimientos de glucosa, por lo que se realizó pancreatectomía subtotal. El informe histo- lógico reveló hiperplasia difusa de células $ß$. En el postoperatorio, persistió hiperinsulinismo (insulinemia $10,8 \mu \mathrm{UI} / \mathrm{mL} /$ glicemia $34 \mathrm{mg} / \mathrm{dl}$ ), que respondió a alimentación fraccionada y dosis bajas de diazóxido, con buena tolerancia y sin presentar reacciones adversas. La evaluación neurológica y EEG al egreso fue normal. A los 14 meses reinició hiperinsulinismo, no controlándose con dieta y diazóxido, por lo que se completó la pancreatectomía $( \pm 100 \%)$, a pesar de lo cual persistió hiperinsulinismo durante 4 meses, haciéndose diabético posteriormente. Presentó trastornos de la deglución que obligaron a alimentación parcial por sonda nasogástrica hasta los 12 meses. Actualmente, a los 3 años 11 meses de edad, su desarrollo psicomotor es normal.

Ambos casos tienen pendiente el resultado del estudio molecular del receptor de sulfonilurea (Baylor College of Medicine, Houston, Texas, USA).

\section{DISCUSIÓN}

El hiperinsulinismo congénito es la causa más común de hipoglicemia persistente en el período neonatal, generalmente refractaria al tratamiento médico ${ }^{1}$. En los últimos años se han realizado importantes avances en el conocimiento de esta entidad, quedando en evidencia su heterogeneidad tanto clínica, histológica, como genética ${ }^{1,3,9-13}$.

La heterogeneidad clínica incluye variabilidad en la edad de inicio de la hipoglicemia, su severidad y respuesta al tratamiento médico. Los $\mathrm{RN}$ con hiperinsulinismo generalmente son macrosómicos, sin embargo, puede presentarse en $\mathrm{RN}$ con peso de nacimiento normal o incluso bajo $5,14,15$.

La hipoglicemia que induce el HNP, habitualmente es severa, $50 \%$ debuta con un cuadro convulsivo, como nuestro paciente $2^{7}$, aunque puede ser detectada por una glicemia de rutina en todo niño con factor de riesgo, como ocurrió en el primer paciente, por tratarse de un RN macrosómico $^{1,15}$. La hipoglicemia es de ayuno y post prandial, habitualmente requiere aportes de glucosa iv superiores a $10 \mathrm{mg} / \mathrm{kg} / \mathrm{min}$ para lograr euglicemia; en general, se presenta como hipoglicenia neonatal persistente, aunque ocasionalmente puede ser transitoria y reaparecer ante 
situaciones de estrés o debutar después del año de edad $1,15,16$.

Actualmente, al existir técnicas sensibles para determinar insulina, basta tener insulina detectable en presencia de hipoglicemia, para hacer diagnóstico de hiperinsulinismo. Otros criterios son: rápida respuesta de la hipoglicemia a la administración de glucagón y niveles bajos de ácidos grasos y cetonas en plasma y orina. Este último criterio, refleja la inapropiada supresión de la lipólisis y cetogénesis por el hiperinsulinismo $1,15,16$.

Histológicamente se describen dos formas, una caracterizada por hiperplasia adenomatosa pancreática difusa y otra con compromiso focal, como lo descrito en las pacientes 1 y 2 , respectivamente. El diagnóstico diferencial entre ellas, permitiría decidir la extensión de la pancreatectomía, en caso de ser necesaria ${ }^{3,9-11}$.

Para diferenciar las formas de HNP, Stanley proponía evaluar la respuesta aguda de insulina a infusión con glucosa, calcio y tolbutamida, método que inicialmente se mostraba promisorio en la diferenciación de ambas formas, pero publicaciones recientes ponen en duda su utilidad ${ }^{12,13,18}$. En la actualidad, los métodos diagnósticos de elección para diferenciar las formas focales de las difusas, serían la estimulación selectiva de arteria pancreática con calcio, midiendo la respuesta de insulina en muestra obtenida de la vena hepática y la cateterización preoperatoria de vena porta y hepática, obteniéndose muestras selectivas de la cabeza, cuerpo y cola del páncreas, para determinar niveles de insulina, glicemia y péptido $\mathrm{C}^{19}$. Estas técnicas sólo se realizan en centros altamente especializados ${ }^{7,16}$. Cabe destacar que 2/3 de las formas focales se encuentran en la cabeza del páncreas, zona que habitualmente se preserva en la pancreatectomía subtotal.

No existe consenso respecto de los criterios morfológicos de hiperinsulinismo. Para algunos autores, sólo es posible diferenciar histológicamente las formas focales de las difusas, combinando la microscopia convencional con histomorfometría y técnicas de inmunocitoquímica ${ }^{9-11}$. En nuestros casos, a pesar de que sólo se realizó microscopia convencional e inmunohistoquímica para insulina, hubo concordancia en los informes respecto de las formas difusa o focal con la evolución clínica de los pacientes. Sin embargo, estos informes se realizaron posterior a las pan- createctomías, lo que no benefició al paciente portador de la forma focal, cuyas alteraciones sólo comprometían la cabeza del páncreas.

En relación a la herencia y bases moleculares del HNP, se ha demostrado que aproximadamente $50 \%$ de los casos son familiares, encontrándose mutaciones en 4 genes relacionados con la secreción de insulina (Figura 1). Se pueden clasificar en cinco entidades: a) mutación autosómica recesiva del canal de K-APT; b) mutación autosómica dominante del canal de K-APT; c) alteración focal del canal de K-APT; d) mutación autosómica dominante del gen de glucokinasa; e) mutación autosómica dominante del gen de glutamato dehidrogenasa ${ }^{3,8,18,20,21}$.

Determinar las bases moleculares del hiperinsulinismo permitiría realizar el consejo genético y orientar la decisión terapéutica; por ejemplo cuando la mutación está en el canal de K-APT, se sabe que no responden al uso de diazóxido ${ }^{16}$, como podría ser el caso de la segunda paciente.

Las formas difusas son de herencia autosómica recesiva y en la mayoría de los casos se deben a mutaciones en los genes que codifican las 2 subunidades de los canales de K-APT dependientes de la célula ß: el gen SUR-1, que codifica el receptor de la sulfonilurea y el gen KIR6.2. Ambos genes se ubican en la región p15.1 del cromosoma 11. Estas mutaciones producen depolarización permanente de la célula $\beta^{3,10}$. Recientemente se han descrito mutaciones en el gen de la glutamato dehidrogenasa, que produce hiperinsulinismo asociado a hiperamonemia ${ }^{20,21}$. Este cuadro, generalmente es gatillado por el aumento de aminoácidos en la dieta. Existen además algunos reportes de mutaciones en el gen de la glucokinasa, lo que alteraría el sensor de la concentración de glucosa sanguínea 22 .

Las formas focales se presentan en forma esporádica y se las ha relacionado con la pérdida de la contribución del alelo materno del gen SUR1 de la región sometida a impronta. El cromosoma materno está implicado en la expresión de genes que regulan el ciclo celular, su pérdida lleva a la sobreexpresión de genes paternos que estimulan la producción del factor estimulador insulino-simil tipo 2, lo que induciría una proliferación anormal de las células $\beta$ afectadas 3,8 .

Por último, en relación al manejo, cabe destacar que dada la severidad de la hipoglicemia que 


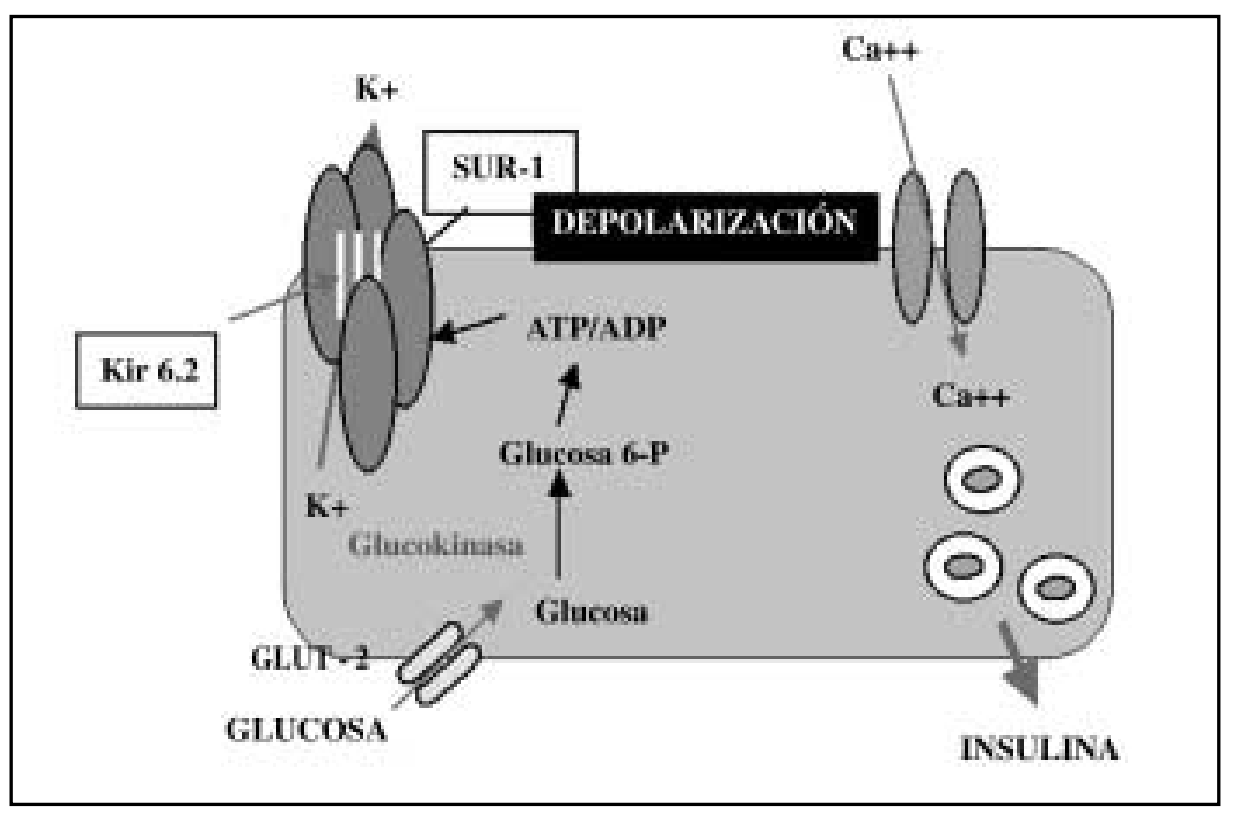

Figura 1. Mecanismos de regulación de la célula ß pancreática. La glucosa circulante es regulada primariamente por la insulina secretada por la célula ß pancreática. En condiciones de reposo la membrana de la célula ß se mantiene hiperpolarizada debido a la bomba $\mathrm{Na}+\mathrm{K}+$ ATPasa de los canales de potasio $(\mathrm{K}+$ ) ATP sensibles. Estos canales están formados por dos subunidades SUR-1 y KIR 6.2. Cuando los niveles de glucosa aumentan, ésta ingresa a la célula a través del transportador específico GLUT-2, la glucosa es fosforilada por la enzima glucokinasa, aumentando los niveles de ATP. Los niveles de ATP pueden verse incrementados por el metabolismo de otros sustratos, como los aminoácidos entre ellos el glutamato, vía glutamato deshidrogenasa (GDH). El aumento de ATP causa el cierre en los canales K-ATP, lo que produce depolarización de la membrana celular y apertura de canales de calcio dependientes de voltaje y por tanto un aumento en la concentración de calcio intracelular, lo que estimula la secreción de insulina4. Adaptado de: Glaser B, Thornton P, Otonkoski T, Junien C. Genetics of neonatal hyperinsulinism. Arch Dis Child Fetal Neonatal 2000; 82: F79-F86.

induce el HNP, el manejo terapéutico debe ser agresivo, administrando glucosa iv, que sumado al aporte oral, mantenga niveles de glicemia $>60$ $\mathrm{mg} / \mathrm{dL}$; si éstos son inestables se debe asociar glucagón ${ }^{16}$. Concomitantemente, debe iniciarse el tratamiento farmacológico del hiperinsulinismo (Tabla 1), siendo la droga de elección el diazóxido combinado con hidroclorotiazida ${ }^{7,16}$. Otra droga de uso más reciente es el análogo de somatostatina, Octreótide ${ }^{\circledR}$, que además de disminuir el hiperinsulinismo, frena la secreción de múltiples hormonas, lo que sumado a su alto costo, limita su uso como primera elección 7,16,23.

Los niños que no responden al tratamiento médico, deben ser trasladados a un centro médico-quirúrgico especializado para una cirugía precoz. La indicación absoluta de cirugía es en aquellos pacientes en que a pesar de alimentación fraccionada y terapia farmacológica, tengan dependencia de glucosa (enteral o parenteral) para mantener glicemias sobre $60 \mathrm{mg} / \mathrm{dL}^{7,16}$. Idealmente, se debe discriminar si se trata de una forma difusa o focal y así determinar la extensión de la pancreatectomía, ya que el riesgo de desarrollar diabetes en pacientes sometidos a pancreatectomía es cercano a $95 \%{ }^{24}$.

En resumen, el enfrentamiento a un paciente con hiperinsulinismo persistente debe ser precoz y agresivo, tanto en el manejo de la hipoglicemia como en la búsqueda del diagnóstico etiológico. El estudio debiera orientarse a discriminar las formas difusas de las focales y en aquellos hiperinsulinismos refractarios al tratamiento médico, programar el tipo de cirugía más adecuada. 
Tabla 1.

\begin{tabular}{|c|c|c|c|}
\hline Fármaco & Mecanismo de Acción & Dosis & $\begin{array}{l}\text { Reacciones } \\
\text { Adversas }\end{array}$ \\
\hline Diazóxido & $\begin{array}{l}\text { Activa canales K-ATP } \\
\uparrow \text { secreción de adrenalina } \\
\uparrow \text { Gluconeogénesis }\end{array}$ & $\begin{array}{l}5-20 \mathrm{mg} / \mathrm{kg} / \mathrm{día} \\
\text { p.o. cada } 8 \mathrm{~h}\end{array}$ & $\begin{array}{l}\text { Hipertricosis } \\
\text { Hiperuricemia } \\
\text { Hipotensión } \\
\text { Leucopenia } \\
\text { Trombocitopenia } \\
\text { Retención hídrica }\end{array}$ \\
\hline Hidroclorotiazida & $\begin{array}{l}\text { Actúa sinérgicamente con } \\
\text { Diazoxide en apertura de } \\
\text { canales K-ATP }\end{array}$ & $\begin{array}{l}\text { 7-10 mg/kg/día } \\
\text { p.o. cada } 12 \mathrm{~h}\end{array}$ & $\begin{array}{l}\text { Hiponatremia } \\
\text { Hipokalemia }\end{array}$ \\
\hline $\begin{array}{l}\text { Nifedipino } \\
\text { Escasa experiencia }\end{array}$ & $\begin{array}{l}\text { Antagonista de canales de } \\
\text { calcio } \\
\text { Inhibe liberación de insulina }\end{array}$ & $\begin{array}{l}\text { 0,25-2,5 mg/ } \mathrm{kg} / \text { día } \\
\text { p.o cada } 8 \mathrm{~h}\end{array}$ & Hipotensión \\
\hline Glucagón & $\begin{array}{l}\text { Aumenta glicogenolisis/ } \\
\text { Neoglucogénesis }\end{array}$ & $\begin{array}{l}1-10 \mu \mathrm{g} / \mathrm{kg} / \mathrm{h} \text { iv } \\
1 \mathrm{mg} / \mathrm{dosis} \mathrm{im} / \mathrm{iv}\end{array}$ & $\begin{array}{l}\text { Náuseas, vómitos } \\
\uparrow \text { GH } \\
\uparrow \text { Contractilidad } \\
\text { miocárdica } \\
\downarrow \text { enzimas gástricas } \\
\text { y pancreáticas }\end{array}$ \\
\hline $\begin{array}{l}\text { Agonista } \\
\text { de somatostatina }\end{array}$ & $\begin{array}{l}\text { Activa proteína } \mathrm{G} \text { acoplada al } \\
\text { canal de } \mathrm{K} \\
\text { Inhibe liberación de Insulina }\end{array}$ & $\begin{array}{l}5-20 \mu \mathrm{g} / \mathrm{kg} / \text { día iv } \\
\text { en infusión iv o sc }\end{array}$ & $\begin{array}{l}\text { Disminuye } \\
\text { secreción } \\
\text { GH, TSH, ACTH } \\
\text { Esteatorrea } \\
\text { Colelitiasis } \\
\text { Distensión } \\
\text { abdominal }\end{array}$ \\
\hline
\end{tabular}

\section{REFERENCIAS}

1. Stanley CA. Hiperinsulinism in infants and children. Pediatr Clin North Am 1997; 44: 363-74.

2. Menni F, De Lonlay-Debeney P, Sevin C, Tovati G, Peigne C, Barbier V et al. Neurologic outcomes of 90 neonates and infants with persistent hyperinsulinemic hypoglicemia. Pediatr 2001; 107: 476-9.

3. Glaser B, Thornton P, Otonkoski T, Junien C. Genetics of neonatal hyperinsulinism. Arch Dis Child Fetal Neonatal 2000; 82: F79-F86.

4. Shilyanski J, Fisher S, Cutz E, Perlman K, Filer RM. Is the $95 \%$ pancreatectomy the procedure of choice for treatment of persistent hyperinsulinemic hypoglicemia of the neonate? J Pediatr Surg 1997; 32: 342-6.
5. Asenjo S, Gieisner A, Wilhelm V, Salas J, Treuer S, Rojas S ET aL. Hiperinsulinismo en recién nacido. Rev Chil Pediatr 1986; 57: 564-7.

6. Rahier J, Guiot Y, Sempox C. Persistent hyperinsulinaemic hypoglycaemia of infancy: a heterogeneous syndrome unrelated to nesidioblastosis. Arch Dis Child Fetal Neonatal 2000; 82: F108F112.

7. De Lonlay-Debeney P, Poggi-Travert F, Fournet J, Sempoux $\mathrm{CH}$, Dionisi C, Brunele F et al. Clinical features of 52 neonates with hyperinsulinism. N Engl J Med 1999; 340: 1169-75.

8. Sempoux $\mathrm{CH}$, Guiot Y, Rahier J. The focal form of persistent hyperinsulinemic hypoglycemia of infancy. Diabetes 2001; 50: S182-S183. 
9. Sempoux C, Guiot Y, Lefevre A, Nihoul-Fékété C, JaUBERT F, SAUdubray M et al. Neonatal hyperinsulinemic hypoglicemia: heterogeneity of the syndrome and keys for differential diagnosis. J Clin Endocrinol Metab 1998; 83: 1455-61.

10. Sempoux C, Guiot Y, Dubois D, Nolevaudx C, Saudubray J-M, Nihoul-Férété C et al. Pancreatic Bcell proliferation in persistent hyperinsulinemic hypoglycemia of infancy: An inmunohistochemical study of 18 cases. Mod Pathol 1998; 11: 444-9.

11. Reinecke-LÜthge A, Koschoreck F, KLöppel G. The molecular basis of persistent hyperinsulinemic hypoglycemia of infancy and its pathologic substrates. Virchows Arch 2000; 436: 1-5.

12. Straub S, Cosgrove K, ÄmmäLä C, Shepherd R, O’Brien E, Barnes Ph et al. Hyperinsulinism of Infancy. The regulated release of insulin by $\mathrm{K}$ ATP chanel-independent pathways. Diabetes 2001; 50: 329-39.

13. Grimberg A, Ferry R, Keluy A, Koo-McCoy S, PoLONSKy K, GLaser B ET al. Dysregulation of insulin secretion in children with congenital hyperinsulinism due to sulfonylurea receptor mutations. Diabetes 2001; 50: 322-8.

14. AKINBI H, GERDES J. Macrosomic infants of nondiabetic mothers and elevated C-peptide levels in cord blood. J Pediatr 1995; 127: 481-4.

15. LTEIF A, SCHWENK WF. Hypoglycemia in infants and children. Endocrinol and Metab Clin 1999; 28: 619-44.

16. Aynsley-Green A, Hussain K, Saudubray JM, NuhoulFékété C, De Lonlay-Debeney P, Brunele $F$ et al. Practical management of hyperinsulinism in infancy. Arch Dis Child Fetal Neonatal 2000; 82: F98-F107.

17. Jä̈̈skeläInen J, Huopio $H$, Komulainen J, Miettinen R, KäRKKäINEN P, LAAKSO M ET AL. Acute Insulin Response test for the Differential Diagnosis of
Congenital Hyperinsulinism. J Clin Endocrinol Metab 2002; 87: 4502-7.

18. Thornton P, Mac Mulen C, Ganguly A, Ruchew E, SteinkRauss L, CRANe A et al. Clinical and molecular characterization of a dominant form of congenital hyperinsulinism caused by a mutation in the high affinity sulfonylurea receptor. Diabetes 2003; 52: 2403-10.

19. Staniey Ch, Thornton P, Ganguly A, MacMulen $C$, Underwood P, Bhatia P et al. Preoperative evaluation of infants with focal or diffuse congenital hyperinsulinism by intravenous acute insulin response test and selective pancreatic arterial calcium stimulation. J Clin Endocrinol Metab 2004; 89: 288-96.

20. Mac Mulen C, Fang I, Hsu B, Kelly A, De LonlayDebeney P, Saudubray J-M et al. Hyperinsulinism/ hyperammonemia syndrome in children with regulatory mutations in the inhibitory guanosine triphosphate-binding domain of glutamate dehydrogenase. J Clin Endocrinol Metab 2000; 86: 1782-7.

21. Kelly A, Ng D, Ferry Jr R, Grimberg A, Koo-McCoy $\mathrm{S}$, Thornton $\mathrm{P}$ et aL. Acute insulin responses to leucine in children with the hyperinsulinism/ hyperammonemia syndrome. J Clin Endocrinol Metab 2001; 86: 3724-8.

22. Glaser B, Kesovan P, Heyman M, Davis E, MB, BS et AL. Brief report: Familial hyperinsulinism caused by an activating Glucokinase mutation. $\mathrm{N}$ Engl J Med 1998; 338: 226-30.

23. Thornton P, Alter C, Levitt L, Baker L, Stanley Ch. Short and long-term use of octreotide in the treatment of congenital hyperinsulinism. J Pediatr 1993; 123: 637-43.

24. Labrune P, Lechevamer S, Rault M, Odiève M. Diabetes Mellitus 14 years after subtotal pancreatectomy for neonatal hyperinsulinism. J Pediatr Surg 1990; 25: 1246-7. 